\title{
Evaluation of Endothelial Function and Insulin Sensitivity in Patients with Prolactinoma
}

\section{F. Buket BAYRAM ${ }^{1}$, Bahar TEKIN ${ }^{1}$, Dilek GOGAS YAVUZ ${ }^{2}$}

${ }^{1}$ Department of Internal Medicine, Marmara University Hospital, Marmara University School of Medicine, İstanbul, Turkey

${ }^{2}$ Department of Internal Medicine, Section of Endocrinology and Metabolism, Marmara University Hospital, Marmara University School of Medicine, İstanbul, Turkey

Objective: Hyperprolactinemia has been reported to be associated with endothelial dysfunction, abnormalities of carbohydrate metabolism and insulin sensitivity. The aim of this study was to evaluate carbohydrate metabolism and endothelial function of our patients in outpatient clinics.

Material and methods: In this cross-sectional study, currently or previously treated 87 hyperprolactinemic patients with PRL-secreting adenoma (fifty four with microadenoma and 33 with macroadenoma; 21 men, 66 women; aged $39,9 \pm 8,8$ years) and 55 healthy controls (twelve men, 43 women; aged $38.8 \pm 10$ years) of comparable gender and age with normal PRL values were included. Anthropometric parameters (body mass index (BMI), blood pressure and waist circumference) and serum glucose, insulin, lipid profiles were measured. Carotid intima media thicknesses (CIMT) were measured by using GE Logic 3 Expert Doppler Ultrasonography to evaluate endothelial function. Insulin resistance was calculated by using homeostasis model assessment of insulin resistance (HOMA IR) and insulin sensitivity index (ISI) Matsuda.
Results: No significant differences were observed in systolic and diastolic blood pressure measurements or BMI values between prolactinoma and control group $(>p=0,05)$. Serum lipid profile levels and waist circumference measurements were also similar, compared to control group (Lowdensity lipoprotein ( $L D L)(p=0,42)$, triglycerides (TG) $(p=0,15)$, high-density lipoprotein (HDL) $(p=0,48)$, total cholesterol $(p=0,17)$ and waist circumference $(p=0,94))$. There were no significant differences in CIMT values, HOMA and Matsuda index scores between prolactinoma and control groups (respectively $(p=0,47),(p=0,68),(p=0,54))$.

Conclusion: In contrast to previous studies, BMD and CIMT measurements, insulin resistances and serum lipid levels in prolactinoma and control groups were similar in our study. This can be associated with that most of our patients are under treatment or were previously treated. Although our study results support the opinion that treatment with dopaminergic agents could improve BMD, endothelial function and insulin sensitivity in prolactinoma patients. 